\title{
Bifurcations of a controlled two-bar linkage motion with considering viscous frictions
}

\author{
Qingkai Han*, Xueyan Zhao, Xingxiu Li and Bangchun Wen \\ School of Mechanical Engineering and Automation, Northeastern University, Shenyang, China \\ Received 4 February 2010 \\ Revised 2 May 2010
}

\begin{abstract}
In this paper, we investigate the joint viscous friction effects on the motions of a two-bar linkage under controlling of OPCL. The dynamical model of the two-bar linkage with an OPCL controller is firstly set up with considering the two joints' viscous frictions. Thereafter, the motion bifurcations of the two-bar linkage along the values of joint viscous frictions are obtained using shooting method. Then, single-periodic, multiple-periodic, quasi-periodic and chaotic motions of link rotating angles are simulated with given different viscous friction values, and they are illustrated in time domain waveforms, phase space portraits, amplitude spectra and Poincare mapping graphs, respectively. Additionally, for the chaotic case, Lyapunov exponents and hypothesis possibilities of the two joint motions are also estimated.
\end{abstract}

Keywords: Two-bar linkage system, OPCL controller, viscous friction, motion bifurcation, chaos

\section{Introduction}

The unavoidable frictions existing in rotational joints will greatly affect the movements, dynamical characteristics and control efforts of many mechanical systems. Moreover, friction properties of a mechanical system inevitably have a great influence on the control efforts [1-3]. A two-bar linkage with simple mechanism structure but highly coupled nonlinearity, which can achieve various motions with different dynamical characteristics, is critical for analyzing mechanical movement and controlling behaviors. Especially, investigation of the complicated chaotic motions of such a system are often very important to help improve its working performance, optimize its structure parameters and develop new control methods in many situations. Moreover, in order to improve the practical dynamical properties of the controlled mechanical system, the study of effects of the existing joint frictions of the controlled structure is also impending.

Nowadays, there exist many control strategies for a two-bar linkage to achieve various patterns of motions. Some controllers based on artificial neural networks (ANN controllers) for achieving both the small swing and giant rotating motions has been implemented successfully [4,5]. While, chaotic motions of a two-link mechanism can be obtained via PD feedback control and model feedback control [6,7]. The motion bifurcation properties of a two-link mechanism also were analyzed by [8]. The transferring process of a two-link mechanism from period-doubling bifurcation to chaotic motions was addressed based on changing control variables in [9]. The open-plus-close-loop (OPCL) control strategy, which was recently developed for the complex dynamic systems [10-12], was used to achieve the controlled synchronization for both the small swing and giant rotating motions of a two-bar linkage by authors [13]. Even with the influences of uncertainty structure parameters and environments, unknown loads, frictions and so on, the synchronization control strategy for a robot system could be realized by designing proper controllers in order to make the synchronization errors converge to zero [14,15].

${ }^{*}$ Corresponding author: Qingkai Han, School of Mechanical Engineering and Automation, Northeastern University, Shenyang, 110189, China. Tel.: +86 24 83680545; Fax: +8624 83679731; E-mail: qhan@mail.neu.edu.cn. 


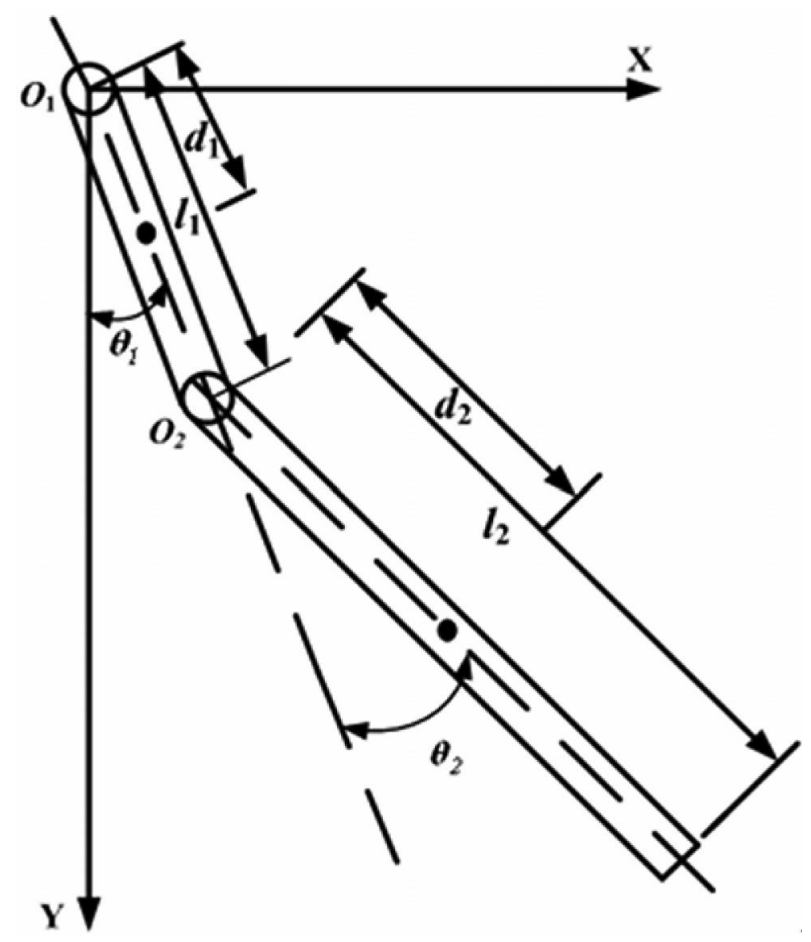

Fig. 1. Model of a two-bar linkage.

In this paper, a controlled two-bar linkage is studied with especially considering its joint viscous frictions. Firstly, the dynamical model of the two-bar linkage with OPCL controller is established involving the joint viscous friction. Then, the motion bifurcations of the rotating joint angles with respect to the joint viscous frictions are obtained using shooting method. Referring to the obtained bifurcation diagrams, some typical motions of each joint are numerically simulated under different friction values, and they are demonstrated with time domain waveforms, frequency spectra, phase space portraits and Poincare mapping graphs, respectively. In addition, the maximum Lyapunov exponents and surrogate data method are also used to describe the chaotic motions under given control parameters and friction coefficients. Finally, the conclusions are made.

\section{Model of the two-bar linkage with OPCL controller and joint friction}

\subsection{Dynamic equations of the two-bar linkage}

A two-bar linkage is shown in Fig. 1. The upper link (Link 1) and the lower link (Link 2) can rotate respectively around their joints of $o_{1}$ and $o_{2}$ within the range of $[-\pi, \pi]$. The joint $o_{1}$ is fixed on the ground. In the coordinate system of $\mathrm{O}_{1} \mathrm{xy}, \theta_{1}$ is the rotational angle of Link 1 with respect to y axis, and $\theta_{2}$ is the rotational angle of Link 2 with respect to the centerline of Link 1 .

The dynamic equation of the two-bar linkage mechanism is given as follows,

$$
\left[\begin{array}{ll}
M_{11} & M_{12} \\
M_{21} & M_{22}
\end{array}\right]\left\{\begin{array}{l}
\ddot{\theta}_{1} \\
\ddot{\theta}_{2}
\end{array}\right\}+\left\{\begin{array}{l}
C_{1}\left(\theta_{1}, \theta_{2}, \dot{\theta}_{1}, \dot{\theta}_{2}\right) \\
C_{2}\left(\theta_{1}, \theta_{2}, \dot{\theta}_{1}, \dot{\theta}_{2}\right)
\end{array}\right\}+\left\{\begin{array}{l}
K_{1}\left(\theta_{1}, \theta_{2}\right) \\
K_{2}\left(\theta_{1}, \theta_{2}\right)
\end{array}\right\}=\left\{\begin{array}{l}
\tau_{1} \\
\tau_{2}
\end{array}\right\}-\left\{\begin{array}{l}
f_{1} \\
f_{2}
\end{array}\right\}
$$

where $f_{1}, f_{2}$ are friction forces coming from the two joints of the two-bar linkage, and they are stated in following section. 


$$
\begin{aligned}
& M_{11}=m_{1} d_{1}^{2}+m_{2}\left(l_{1}^{2}+d_{2}^{2}+2 l_{1} d_{2} \cos \theta_{2}\right)+I_{1}+I_{2} \\
& M_{21}=m_{2}\left(d_{2}^{2}+l_{1} d_{2} \cos \theta_{2}\right)+I_{2} \\
& M_{12}=m_{2}\left(d_{2}^{2}+l_{1} d_{2} \cos \theta_{2}\right)+I_{2} \\
& M_{22}=m_{2} d_{2}^{2}+I_{2} \\
& C_{1}\left(\theta_{1}, \theta_{2}, \dot{\theta}_{1}, \dot{\theta}_{2}\right)=-m_{2} l_{1} d_{2} \dot{\theta}_{2}^{2} \sin \theta_{2}-2 m_{2} l_{1} d_{2} \dot{\theta}_{1} \dot{\theta}_{2} \sin \theta_{2} \\
& C_{2}\left(\theta_{1}, \theta_{2}, \dot{\theta}_{1}, \dot{\theta}_{2}\right)=m_{2} l_{1} d_{2} \dot{\theta}_{1}^{2} \sin \theta_{2} \\
& K_{1}\left(\theta_{1}, \theta_{2}\right)=\left(m_{1} d_{1}+m_{2} l_{1}\right) g \sin \theta_{1}+m_{2} d_{2} g \sin \left(\theta_{1}+\theta_{2}\right) \\
& K_{2}\left(\theta_{1}, \theta_{2}\right)=m_{2} g d_{2} \sin \left(\theta_{1}+\theta_{2}\right)
\end{aligned}
$$

And $m_{i}$ is the mass of Link $i, I_{i}$ is the moment of inertia of Link $i$ with respect to its mass center, $d_{i}$ is the distance between Link $i$ and the joint $i$; $g$ is the acceleration of gravity; $\tau_{i}$ is driving moment on the joint $i, i=1,2$.

\subsection{The OPCL controller and its stability}

If an uncontrolled system is given as the following general formula,

$$
\ddot{x}=F(x, \dot{x}, t)
$$

where $x=\left\{x_{1}, x_{2}, \cdots, x_{n}\right\}^{T}$ is the state variable of the uncontrolled system.

For the two-bar linkage of Eq. (1), the dynamical equations can be transformed into the same form as Eq. (2) as follows

$$
\left\{\begin{array}{l}
\ddot{\theta}_{1} \\
\ddot{\theta}_{2}
\end{array}\right\}=\left[\begin{array}{ll}
H_{11} & H_{12} \\
H_{21} & H_{22}
\end{array}\right]\left\{\begin{array}{l}
R_{1} \\
R_{2}
\end{array}\right\}
$$

where, $\left[\begin{array}{ll}H_{11} & H_{12} \\ H_{21} & H_{22}\end{array}\right]=\left[\begin{array}{ll}M_{11} & M_{12} \\ M_{21} & M_{22}\end{array}\right]^{-1},\left\{\begin{array}{l}R_{1} \\ R_{2}\end{array}\right\}=\left\{\begin{array}{l}\tau_{1}-f_{1}-C_{1}\left(\theta_{1}, \theta_{2}, \dot{\theta}_{1}, \dot{\theta}_{2}\right)-K_{1}\left(\theta_{1}, \theta_{2}\right) \\ \tau_{2}-f_{2}-C_{2}\left(\theta_{1}, \theta_{2}, \dot{\theta}_{1}, \dot{\theta}_{2}-K_{2}\left(\theta_{1}, \theta_{2}\right)\right.\end{array}\right\}$.

To a given system of Eq. (2), a moving goal is defined as

$$
g=\left\{g_{1}, g_{2}, \cdots, g_{n}\right\}^{T}
$$

With the synchronous tracking theory, the control error is defined as follows

$$
e=x-g=\left\{x_{1}-g_{1}, x_{2}-g_{2}, \cdots, x_{n}-g_{n}\right\}^{T}=\left\{e_{1}, e_{2}, \cdots, e_{n}\right\}^{T}
$$

In a small neighborhood of the goal of Eq. (4), the uncontrolled system of Eq. (2) is locally linearized via Taylor expansions as

$$
\begin{aligned}
\ddot{x} & =F(x, \dot{x}, t)=F(g+e, \dot{g}+\dot{e}, t)=F(g, \dot{g}, t)+(\partial F(g, \dot{g}, t) / \partial g) e+(\partial F(g, \dot{g}, t) / \partial \dot{g}) \dot{e}+o^{2}(g, \dot{g}) \\
& =F(g, \dot{g}, t)+J_{g} e+J_{\dot{g}} \dot{e}+o^{2}(g, \dot{g})
\end{aligned}
$$

where, $J_{g}$ and $J_{\dot{g}} s$ are Jacobian matrices of $F(g, \dot{g}, t)$ with respect to $g$ and $\dot{g}$, respectively. Then, an OPCL controller is designed as

$$
U=\ddot{g}-F(g, \dot{g}, t)-J_{g} e-J_{\dot{g}} \dot{e}+A \dot{e}+B e
$$

where, $\ddot{g}-F(g, \dot{g}, t)$ is the open loop component, $-J_{g} e-J_{\dot{g}} \dot{e}+A \dot{e}+B e$ is the close loop component. In Eq. (7), the coefficient matrices of the OPCL controller, $A$ and $B$ are diagonal ones.

The controlled system, composing of the uncontrolled system of Eq. (2) with the above OPCL controller $U$, is re-written as follows

$$
\begin{aligned}
\ddot{x} & =F(x, \dot{x}, t)+U \\
& =F(g, \dot{g}, t)+J_{g} e+J_{\dot{g}} \dot{e}+o^{2}(g, \dot{g})+\ddot{g}-F(g, \dot{g}, t)-J_{g} e-J_{\dot{g}} \dot{e}+A \dot{e}+B e \\
& =\ddot{g}+A \dot{e}+B e+o^{2}(g, \dot{g})
\end{aligned}
$$


Furthermore, after ignoring higher order smaller terms, the tracking error equation of Eq. (5) is also deduced as follows

$$
\ddot{e}=\ddot{x}-\ddot{g}=\mathrm{A} \dot{e}+B e
$$

Namely,

$$
\left\{\begin{array}{c}
\ddot{e}_{1} \\
\ddot{e}_{2} \\
\vdots \\
\ddot{e}_{n}
\end{array}\right\}=\operatorname{diag}\left(a_{11}, a_{22}, \cdots, a_{n n}\right)\left\{\begin{array}{c}
\dot{e}_{1} \\
\dot{e}_{2} \\
\vdots \\
\dot{e}_{n}
\end{array}\right\}+\operatorname{diag}\left(b_{11}, b_{22}, \cdots, b_{n n}\right)\left\{\begin{array}{c}
e_{1} \\
e_{2} \\
\vdots \\
e_{n}
\end{array}\right\}=\left\{\begin{array}{c}
a_{11} \dot{e}_{1}+b_{11} e_{1} \\
a_{22} \dot{e}_{2}+b_{22} e_{2} \\
\vdots \\
a_{n n} \dot{e}_{n}+b_{n n} e_{n}
\end{array}\right\}
$$

From Eq. (10), it can be proved that if $\ddot{e}_{i}=a_{i i} \dot{e}_{i}+b_{i i} e_{i}(i=1,2, \cdots, n)$ is asymptotically stable, the original system of Eq. (8) is asymptotically stable too. Here a Lyapunov function $V$ is defined as

$$
V\left(\dot{e}_{i}, e_{i}\right)=\frac{b_{i i}}{2 a_{i i}} e_{i}^{2}-\frac{1}{2 a_{i i}} \dot{e}_{i}^{2}>0
$$

And we have its differential function as follows

$$
\dot{V}\left(\dot{e}_{i}, e_{i}\right)=\frac{b_{i i} e_{i} \dot{e}_{i}-\dot{e}_{i} \ddot{e}_{i}}{a_{i i}}=\frac{b_{i i} e_{i} \dot{e}_{i}-\dot{e}_{i}\left(a_{i i} \dot{e}_{i}+b_{i i} e_{i}\right)}{a_{i i}}=-\dot{e}_{i}^{2}<0
$$

It is known that the controlled system is asymptotically stable if $A$ and $B$ are constant and with negative real part of eigenvalues, namely $a_{i i}<0$ and $b_{i i}<0$. Therefore, the OPCL controller is effective to be applied on a two-bar linkage to achieve stable motions.

Considering the uncontrolled system of Eq. (1), and setting the state variable as $\theta=x=\left\{x_{1}, x_{2}\right\}^{T}$, i.e. $x_{1}=\theta_{1}$, $x_{2}=\theta_{2}$, the general model of the two-bar linkage system with OPCL controller can be re-written as

$$
\begin{aligned}
\ddot{\theta} & =\ddot{x}+U \\
& =F(x, \dot{x}, t)+U \\
& =F(\theta, \dot{\theta}, t)+U \\
& =\left[\begin{array}{ll}
M_{11} & M_{12} \\
M_{21} & M_{22}
\end{array}\right]^{-1}\left\{\begin{array}{c}
\tau_{1}-C_{1}\left(\theta_{1}, \theta_{2}, \dot{\theta}_{1}, \dot{\theta}_{2}\right)-K_{1}\left(\theta_{1}, \theta_{2}\right) \\
\tau_{2}-C_{2}\left(\theta_{1}, \theta_{2}, \dot{\theta}_{1}, \dot{\theta}_{2}-K_{2}\left(\theta_{1}, \theta_{2}\right)\right.
\end{array}\right\}+U
\end{aligned}
$$

where $\ddot{\theta}$ is the acceleration of the rotational angle of the two-bar linkage, and with the same expression as Eq. (3).

For the two-bar linkage shown in Fig. 1, when only considering the viscous friction, the joint frictions of joint 1 and joint 2, namely, $f_{1}$ and $f_{2}$ in Eq. (1), are simplified as follows [16,17].

$$
f_{1}=f_{v} \dot{\theta}_{1}, \quad f_{2}=f_{v} \dot{\theta}_{2}
$$

where, $f_{v}$ is the viscous friction coefficient. The Eq. (14) will be applied in the following simulations.

It also can be seen that the dynamical system of the two-bar linkage system with OPCL controller, as shown in Eq. (13), contains at least three independent variables, $\theta_{1}, \theta_{2}$ and $U$. From the view point of nonlinearity, it will present complex patterns of motions such as chaos, which will be demonstrated by the following simulations.

\section{Motion bifurcation along the viscous frictions}

Here only the effects of the joint viscous frictions on the joint angle motions are discussed. The goal trajectories of the two joints of the two-bar linkage are designed as

$$
\theta_{1}=\sin (t), \quad \theta_{2}=\sin (t)
$$

The control parameters of Eq. (7) are $A=\operatorname{diag}(-10,-10)$ and $B=\operatorname{diag}(-20,-20)$, respectively. If the joint frictions are completely ignored, namely, $f_{1}=0, f_{2}=0$, the two-bar linkage can achieve synchronous motions, 
Table 1

The dimensionless values of structural parameters of the two-bar linkage

\begin{tabular}{lccccccccc}
\hline Parameters & $m_{1}$ & $m_{2}$ & $I_{1}$ & $I_{2}$ & $l_{c 1}$ & $l_{c 2}$ & $l_{1}$ & $l_{2}$ & $g$ \\
\hline values & 1 & 1 & 0.083 & 0.33 & 0.5 & 1 & 1 & 2 & 9.8 \\
\hline
\end{tabular}

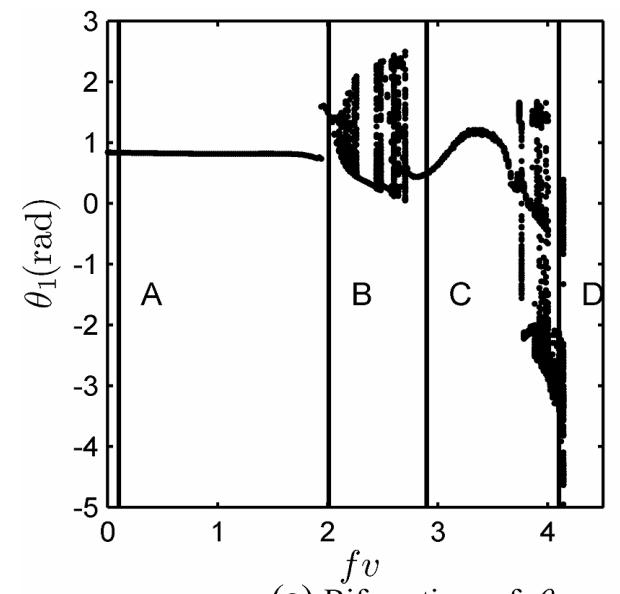

(a) Bifurcations of $\theta_{1}$

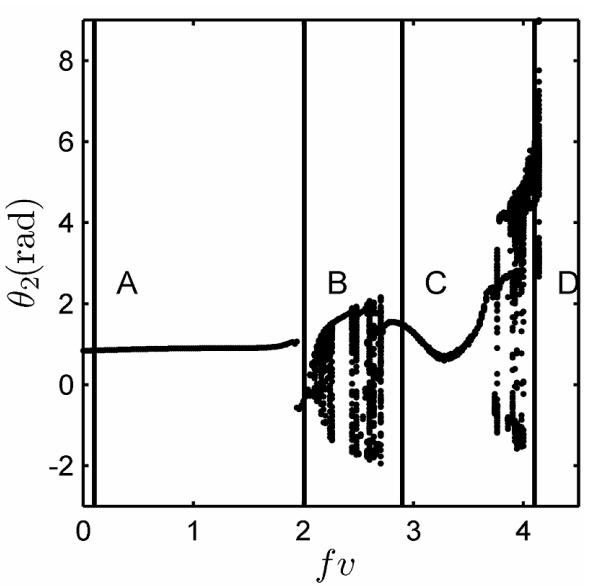

(b) Bifurcations of $\theta_{2}$

Fig. 2. Motion bifurcations along viscous joint friction $f_{v}$ [(a) Bifurcations of $\theta_{1}$, (b) Bifurcations of $\theta_{2}$ ].

in which the angle errors between $\theta_{1}$ and $\theta_{2}$ were within $\left[-0.1 \times 10^{-3}, 0.1 \times 10^{-3}\right]$ and the two angular velocity errors were within $\left[-0.2 \times 10^{-3}, 0.2 \times 10^{-3}\right]$, referring to [13].

In numerical simulations, the dimensionless structural parameters of the two-bar linkage are listed in Table 1.

From numerical simulations, given the initial values of $\theta_{1}, \dot{\theta}_{1}, \theta_{2}$ and $\dot{\theta}_{2}$ as $1,0,1$ and 0 and unchangeable hereon., we can obtain some different types of motions such as single-periodic, multiple-periodic, quasi-periodic and chaotic motions of the two joints when with different values of the viscous joint friction coefficient $f_{v}$ of Eq. (14).

The motion bifurcation diagrams for the two rotating joints are shown in Fig. 2 using the shoot method. The algorithm of the shooting method can be referred as [18]. The bifurcation diagrams are along the bifurcation parameter of $f_{v}$ changing from 0 to 4.15 .

From Fig. 2, it can be seen that $\theta_{1}$ and $\theta_{2}$ display the same bifurcation characteristics obviously. When the value of $f_{v}$ changes between 0 and 2.1, $\theta_{1}$ and $\theta_{2}$ have the periodical characteristic. The jump phenomenon happens at $f_{v}=1.94$. When the value of $f_{v}$ changes between 2.1 and 2.7, $\theta_{1}$ and $\theta_{2}$ appear chaotic characteristics together with periodic motion windows. When the value of $f_{v}$ changes between 2.7 and 3.7, $\theta_{1}$ and $\theta_{2}$ are periodic too. When the value of $f_{v}$ changes between 3.7 and $4.15, \theta_{1}$ and $\theta_{2}$ appear chaotic characteristics again, together with periodic motion windows.

\section{Descriptions of different various motions}

According to the bifurcation diagrams in Fig. 2, the two-bar linkage can achieve various motions if with different viscous friction values.

There are four different cases for the value of $f_{v}$ which is chosen as $0.1,2.01,2.9$ and 4.1 respectively, with respect to the four straight lines noted as A, B, C and D in Fig. 2, respectively. These four motions are listed in the Table 2 and demonstrated in the following sections 4.1-4.4.

\subsection{Single-periodic motions}

The two-bar linkage can achieve single-periodic motions by changing viscous friction coefficient $f_{v}$ referring to the bifurcation diagrams in Fig. 2. The simulation results with the viscous friction coefficient $f_{v}=0.1$ are shown in 
Table 2

The different motions of the two-bar linkage with different friction values

\begin{tabular}{lccccc}
\hline Case No. & & Motion patterns & Poincare mapping & Amplitude spectra & Simulation results \\
\hline A & 0.1 & Single-periodic & One isolated point & Single frequency line \\
B & 2.01 & Multiple-periodic & Isolated points & Several obvious frequency lines & Fig. 4 \\
C & 2.9 & Quasi-periodic & Straight line with isolated points & Several obvious frequency lines & Fig. 5 \\
D & 4.1 & Chaotic & Regular shape with unlimited points & Limited broadband spectra & Fig. 6 \\
\hline
\end{tabular}

Table 3

Lyapunov exponents of the simulated chaos motions

\begin{tabular}{lccccc}
\hline Signal & First order & Second order & Third order & Forth order & Fifth order \\
\hline$\theta_{1}$ & 0.1432 & -0.0375 & -0.0789 & -0.1566 & -2.4472 \\
$\theta_{2}$ & 0.1742 & -0.0287 & -0.0708 & -0.1509 & -2.5306 \\
\hline
\end{tabular}

Fig. 3. The motions of $\theta_{1}$ and $\theta_{2}$ are periodic shown in Fig. 3 (a) and (b). The amplitude spectra of the two angles show that there is only single dominant frequency of about $0.16 \mathrm{~Hz}$ in each of the corresponding amplitude spectra for $\theta_{1}$ and $\theta_{2}$ respectively, as shown in Fig. 3 (c) and (d). Furthermore, both of their phase space portraits, shown in Fig. 3 (e) and (f), are the closed curves, and each of the Poincare mapping portraits is only one isolated point, as shown in Fig. $3(\mathrm{~g})$ and $(\mathrm{h})$. According to these numerical results, it can be concluded that the two-bar linkage moves in stable single-periodic motions in this case, that is, the motions of $\theta_{1}$ and $\theta_{2}$ are periodic.

\subsection{Multiple-periodic motions}

The multiple-periodic motions can be achieved when the viscous friction coefficient $f_{v}=2.04$ as shown in Fig. 4 . It can be seen that the motions of $\theta_{1}$ and $\theta_{2}$ are also periodical but with other harmonic components in Fig. 4 (a) and (b). There are six frequency lines in the corresponding amplitude spectra of $\theta_{1}$ and $\theta_{2}$ respectively as shown in Fig. 4 (c) and (d). Accordingly, both of the phase space portraits, as shown in Fig. 4 (e) and (f), are closed curves, and the Poincare mapping portraits, shown in Fig. 4 (g) and (h), give some isolated points. These indicate that the multi-periodic motion of the two-bar linkage moves stably in this case.

\subsection{Quasi-periodic motions}

The two-bar linkage can also achieve the quasi-periodic motions. The simulation results with respect to the viscous friction coefficient $f_{v}=2.9$ are shown in Fig. 5. From Fig. 5 (a) and (b), it can be seen that the motions of $\theta_{1}$ and $\theta_{2}$ are complex but regular. There are nine and twelve obvious frequency lines for $\theta_{1}$ and $\theta_{2}$ respectively shown in their corresponding amplitude spectra in Fig. 5 (c) and (d). Both of the two phase space portraits, as shown in Fig. 5 (e) and (f), are difficult to distinguish from traditional harmonics and the Poincare mapping portraits of them, shown in Fig. 5 (g) and (h), are the concentrated sticklike areas. These can help to conclude that the simulated motions of the two-bar linkage are quasi-periodic in this case.

\subsection{Chaotic motions}

The two-bar linkage also presents chaotic motions when the viscous friction coefficient $f_{v}=4.1$ shown in Fig. 6 . The simulated responses of $\theta_{1}$ and $\theta_{2}$ are irregular without obvious periods, as shown in Fig. 6 (a) and (b). Their corresponding amplitude spectra as shown in Fig. 6 (c) and (d), demonstrate multi-frequency lines and definite broadband ranges. Moreover, both of the phase space portraits (shown in Fig. 6 (e) and (f)) and the Poincare mapping (shown in Fig. 6 (g) and (h)) of them illustrate irregular shape. According to the qualitative theory of chaos, these motions exhibit chaotic behaviors.

In addition, the obtained chaotic signals shown in Fig. 6 are evaluated via nonlinear analysis methods introduced in [18-21]. The first five order Lyapunov exponents of the time series of $\theta_{1}$ and $\theta_{2}$ are calculated according to algorithm in $[19,20]$ and shown in Table 3. Among them, the maximum Lyapunov exponents for $\theta_{1}$ and $\theta_{2}$ are positive, i.e. 0.1432 and 0.1742 , respectively; and the sum of the rest Lyapunov exponents are both smaller than 

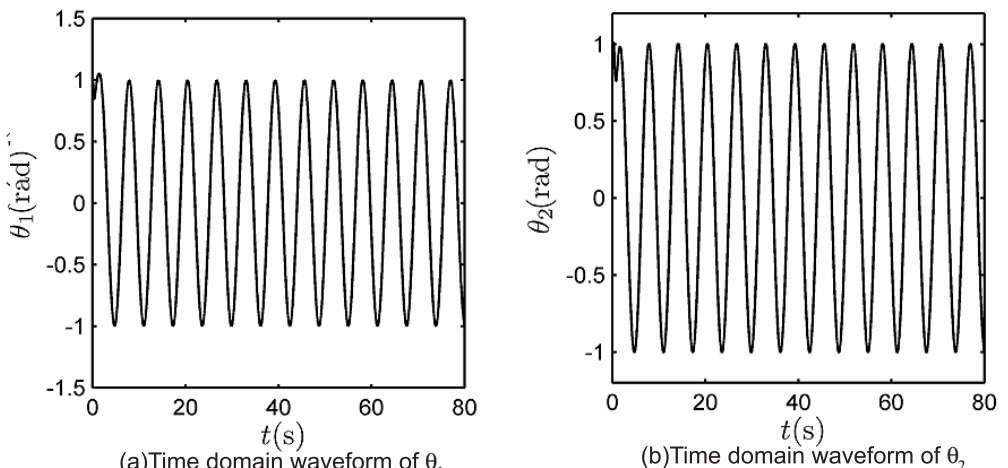

(a)Time domain waveform of $\theta$

(b)Time domain waveform of $\theta_{2}$
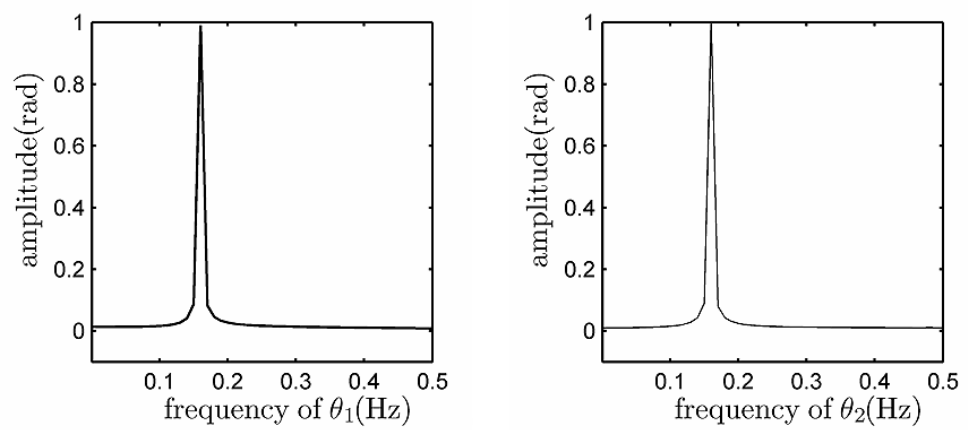

(c)Amplitude spectrum of $\theta_{1}$

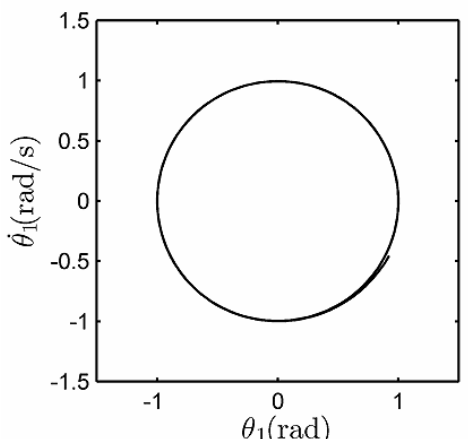

d)Amplitude spectrum of $\theta_{2}$

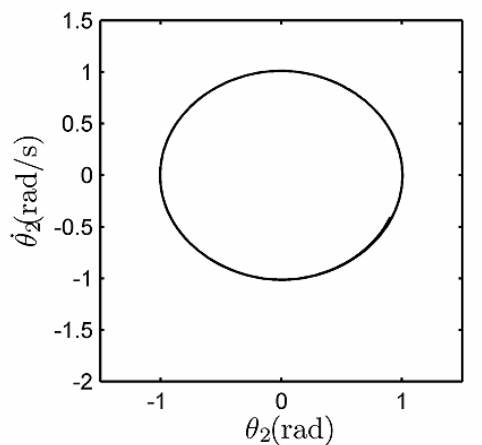

(e)Phase space portrait of $\theta_{1}$
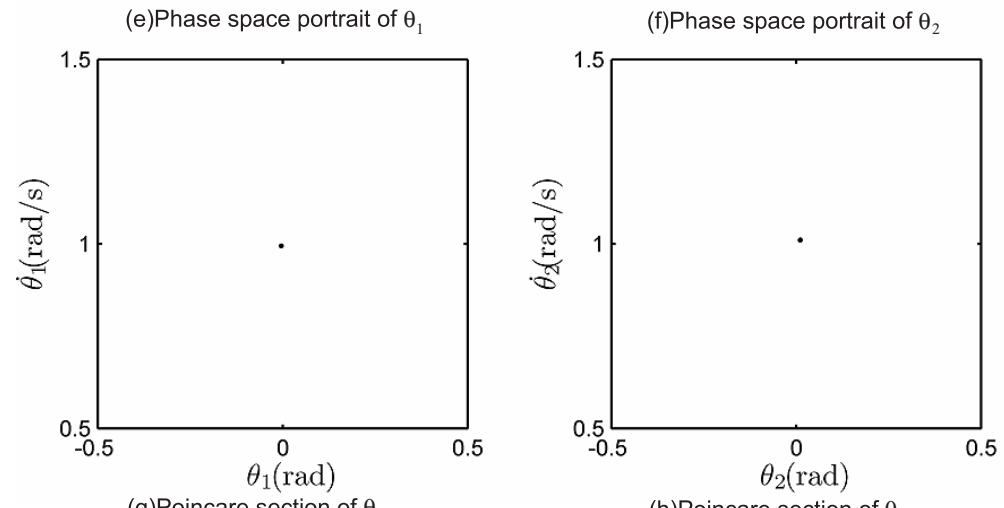

Fig. 3. The simulated single-periodic motions [(a) Time domain waveform of $\theta_{1}$, (b) Time domain waveform of $\theta_{2}$, (c) Amplitude spectrum of $\theta_{1}$, (d) Amplitude spectrum of $\theta_{2}$, (e) Phase space portrait of $\theta_{1}$, (f) Phase space portrait of $\theta_{2}$, (g) Poincare section of $\theta_{1}$, (h) Poincare section of $\left.\theta_{2}\right]$. 

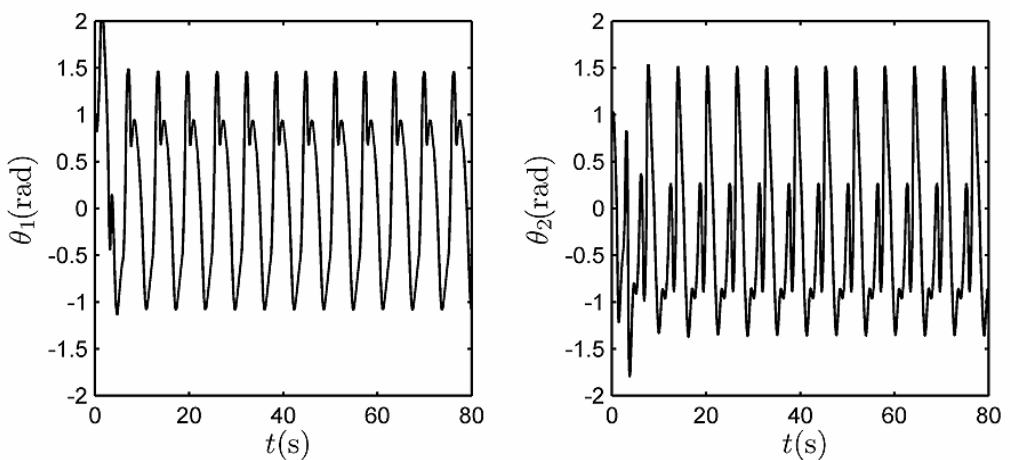

(a)Time domain waveform of $\theta$

(b)Time domain waveform of $\theta$
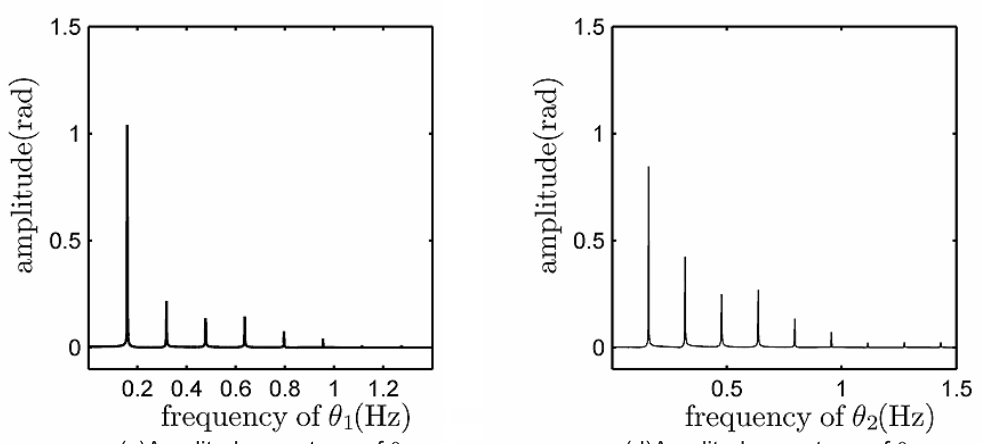

(c)Amplitude spectrum of $\theta$

(d)Amplitude spectrum of $\theta_{2}$
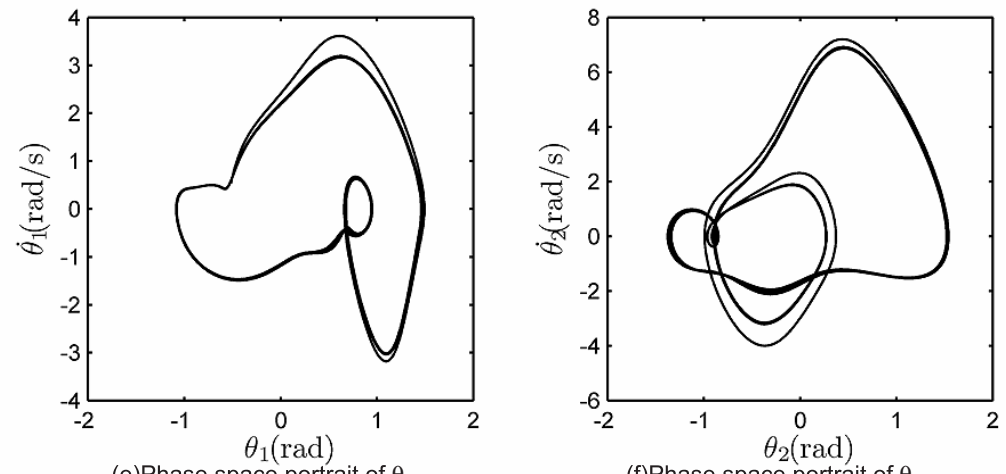

(e)Phase space portrait of $\theta$

(f)Phase space portrait of $\theta_{2}$
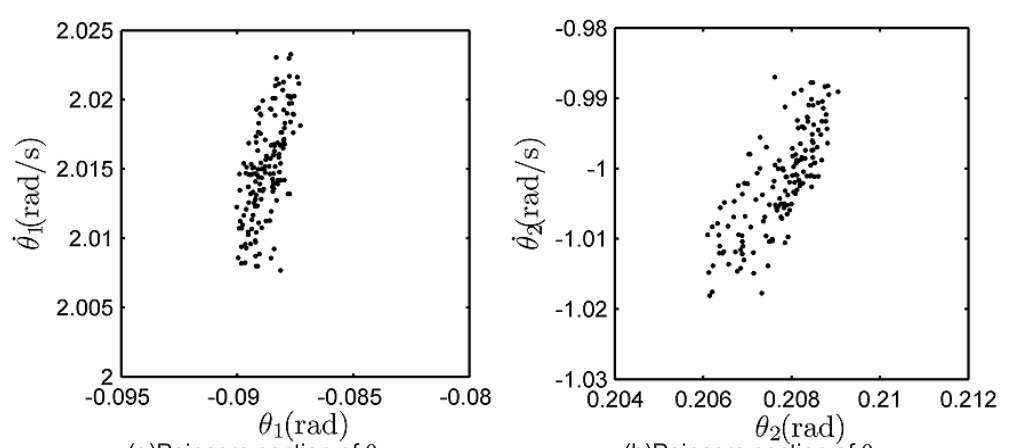

(g)Poincare section of $\theta_{1}$

(h)Poincare section of $\theta_{2}$

Fig. 4. The simulated multiple-periodic motions [(a) Time domain waveform of $\theta_{1}$, (b) Time domain waveform of $\theta_{2}$, (c) Amplitude spectrum of $\theta_{1}$, (d) Amplitude spectrum of $\theta_{2}$, (e) Phase space portrait of $\theta_{1}$, (f) Phase space portrait of $\theta_{2}$, (g) Poincare section of $\theta_{1}$, (h) Poincare section of $\left.\theta_{2}\right]$. 


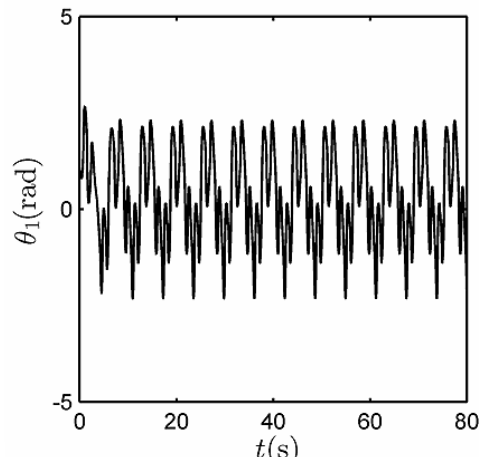

(a)Time domain waveform of $\theta_{1}$
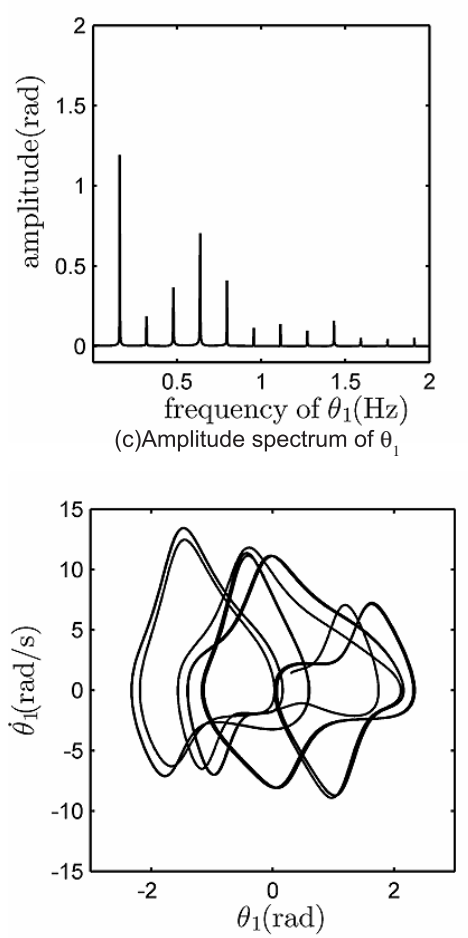

(e)Phase space portrait of $\theta_{1}$

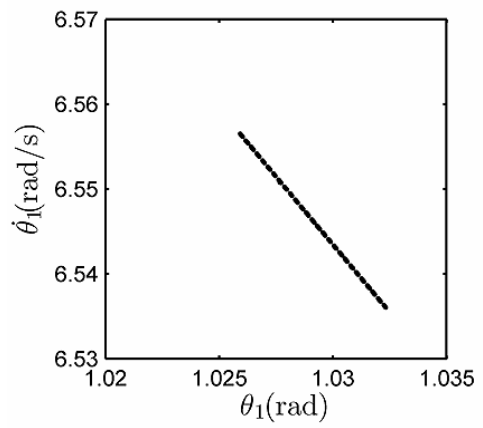

(g)Poincare section of $\theta_{1}$

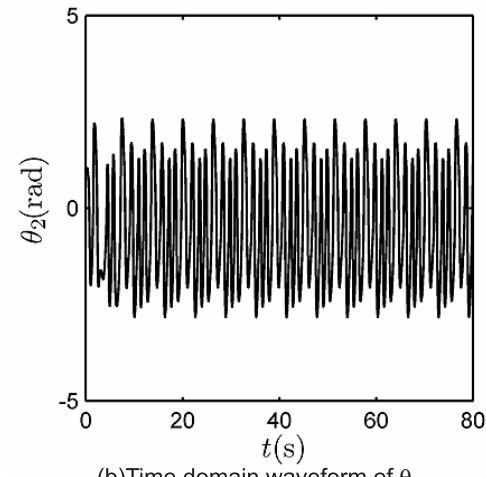

(b) Time domain waveform of $\theta_{2}$

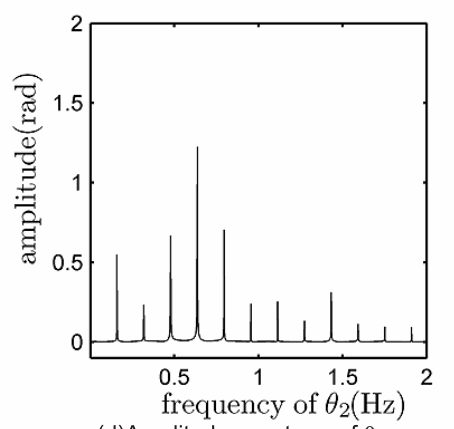

(d)Amplitude spectrum of $\theta_{2}$

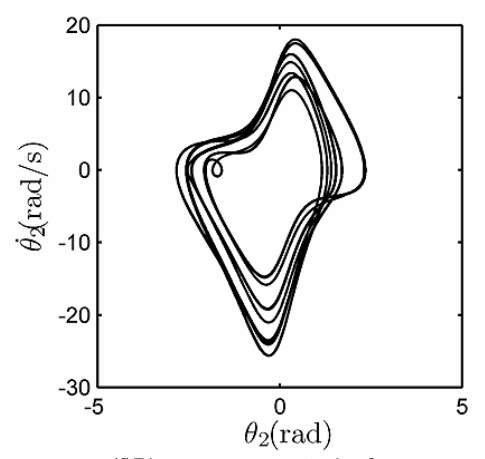

(f)Phase space portrait of $\theta_{2}$

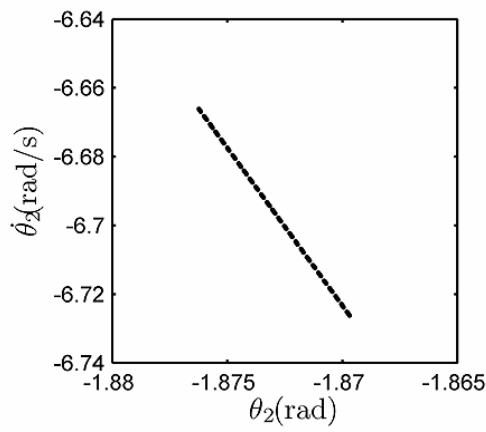

(h)Poincare section of $\theta_{2}$

Fig. 5. The simulated quasi-periodic motions [(a) Time domain waveform of $\theta_{1}$, (b) Time domain waveform of $\theta_{2}$, (c) Amplitude spectrum of $\theta_{1}$, (d) Amplitude spectrum of $\theta_{2}$, (e) Phase space portrait of $\theta_{1}$, (f) Phase space portrait of $\theta_{2}$, (g) Poincare section of $\theta_{1}$, (h) Poincare section of $\left.\theta_{2}\right]$. 


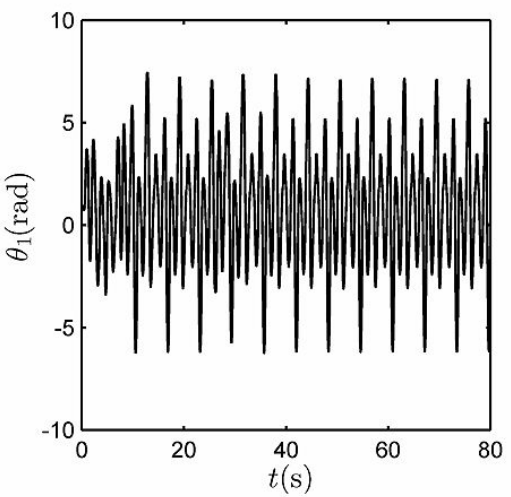

(a)Time domain waveform of $\theta_{1}$
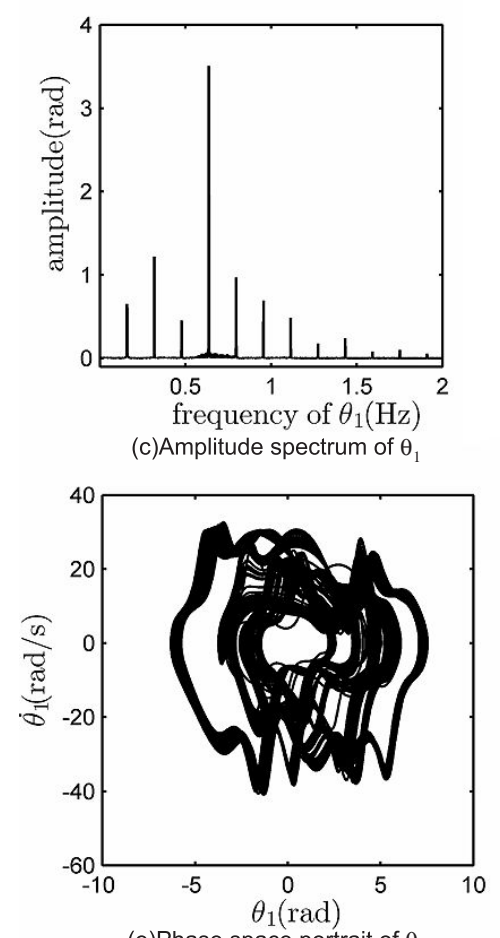

(e)Phase space portrait of $\theta_{1}$

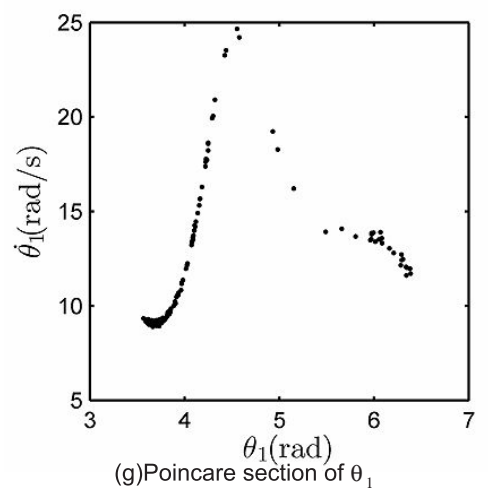

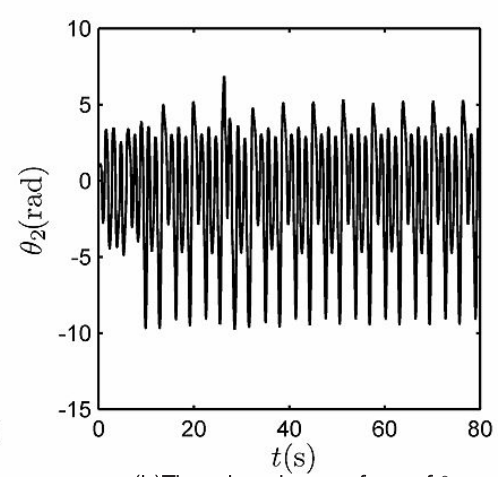

(b)Time domain waveform of $\theta_{2}$

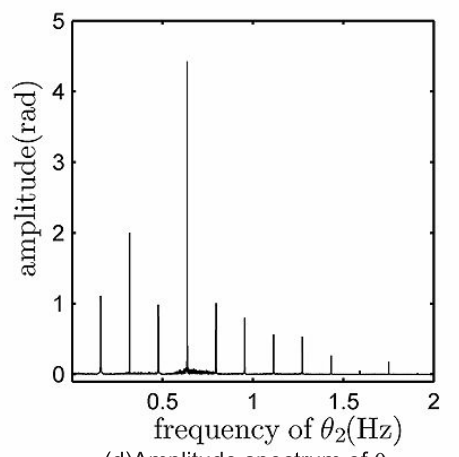

(d)Amplitude spectrum of $\theta_{2}$

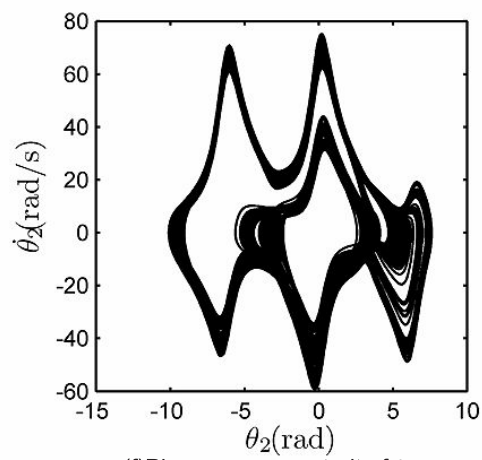

(f)Phase space portrait of $\theta_{2}$

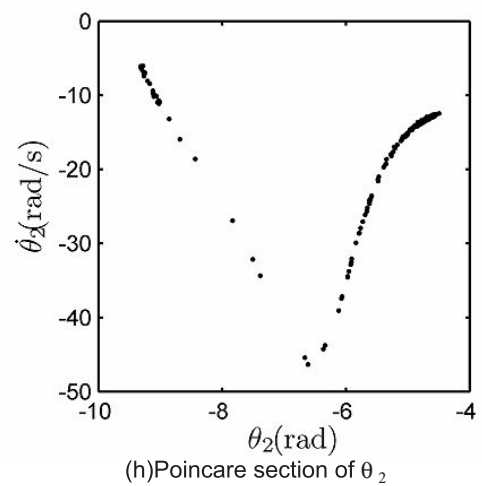

Fig. 6. The simulated chaotic motions [(a) Time domain waveform of $\theta_{1}$, (b) Time domain waveform of $\theta_{2}$, (c) Amplitude spectrum of $\theta_{1}$, (d) Amplitude spectrum of $\theta_{2}$, (e) Phase space portrait of $\theta_{1}$, (f) Phase space portrait of $\theta_{2}$, (g) Poincare section of $\theta_{1}$, (h) Poincare section of $\left.\theta_{2}\right]$. 
0 . The calculated hypothesis possibilities of them with the surrogate data method [21] are $4.2526 \times 10^{-14}$ and $5.9422 \times 10^{-15}$ respectively. The two possibility values are both greatly smaller than 0.05 , an empirical given threshold for chaos. Therefore, it can be seen that the simulated joint rotating angles of $\theta_{1}$ and $\theta_{2}$ in Case D (and shown in Fig. 6) are chaotic.

\section{Conclusions}

The dynamic model of a two-bar linkage considering viscous joint friction and under OPCL control is proposed, where motion bifurcations of it with respect to the viscous friction coefficient are conducted. The numerical simulations show that even if only the viscous friction is considered, the controlled two-bar linkage can act eventually complicated bifurcate into various motions such as periodic, quasi-periodic and chaotic motions.

With different viscous friction coefficients, the two-bar linkage can achieve different motions such as singleperiodic, multiple-periodic, quasi-periodic and chaotic motions, and their motion characteristics are illustrated by the time domain waveform graphs, frequency domain spectra and Poincare mapping graphs clearly.

Furthermore, for the simulated chaotic motions, the calculated maximum Lyapunov exponents of them are positive, and the calculated hypothesis possibilities with the surrogate data method are greatly smaller than 0.05 correspondingly.

\section{Acknowledgements}

The authors gratefully acknowledge that the work was supported by the Key Project of Science and Technology Research Funds of Chinese Ministry of Education (Granted No. 108037).

\section{References}

[1] P.J. Blau, Friction science and technology: from concepts to applications, 2nd Ed, CRC Press, Taylor \& Francis LLC, 2008.

[2] B. Armatrong-Helouvry, P. Dupontand and C. Canudas de Wit, A survey of models, analysis tools and compensation methods for the control of machines with friction, Automatica 30 (1994), 1083-1138.

[3] J. Awrejcewicz and P. Olejnik, Analysis of dynamic systems with various friction laws, Applied Mechanics Reviews 58 (2005), $389-411$.

[4] K. Matsuoka, N. Ohyama, A. Watanabe and M. Ooshima, Control of a giant swing robot using a neural oscillator, Lecture Notes in Computer Science 3611 (2005), 274-284.

[5] Q.K. Han, Z.Y. Qin, X.G. Yang and B.C. Wen, Rhythmic swing motions of a two-link robot with a neural controller, International Journal of Innovative Computing, Information \& Control 3 (2007), 335-342.

[6] L. Shrinivas and A. Ghosal, Possible chaotic motions in a feedback controlled 2R robot, Proceedings of the1996 IEEE, International Conference on Robotics and Automation, Minnesota US, (1996), 1241-1246.

[7] A.S. Ravishankar and A. Ghosal, Nonlinear dynamics and chaotic motions in feedback controlled two- and three-degree-of-freedom robots, The International Journal of Robots Research 18 (1999), 93-108.

[8] F. Verduzco and J. Alvarez, Bifurcation analysis of a 2-DOF robot manipulator driven by constant torques, International Journal of Bifurcation and Chaos 9(4) (1999), 617-627.

[9] K.F. Li, L. Li and Y. Chen, Chaotic motion of a planar 2-dof robot, Machine 29 (2002), 6-8. (in Chinese).

[10] E. Jackson, An open-plus-closed-loop (OPCL) control of complex dynamic systems, Physica D 85 (1995), 1-9.

[11] L.Q. Chen and Y.Z. Liu, A modified open-plus-closed-loop control of chaos in nonlinear oscillations, Physics Letters A 245 (1998), 87-90.

[12] L.Q. Chen, An open-plus-closed-loop control for discrete chaos and hyper chaos, Physics Letters A 281 (2001), $327-333$.

[13] Q.K. Han, X.Y. Zhao and B.C. Wen, Synchronization motions of a two-link mechanism with an improved OPCL method, Applied Mathematics and Mechanics 29 (2008), 1561-1568.

[14] R. Lu, Synchronized trajectory tracking control for parallel manipulators, Doctorial Thesis of University of Toronto, 2006.

[15] H. Nijmeijer and I.M.Y. Mareels, An observer looks at synchronization, IEEE Transactions on Circuits and Systems: Fundamental Theory and Application 44 (1997), 882-890.

[16] H. Olsson, K.J.K.J. Åström, C. Canudas de Wit, M. Gäfwert and P. Lischinsky, Friction models and friction compensation, European Journal of Control 4 (1997), 176-195.

[17] C. Canudas de Wit and J. Carillo, A modified EW-RLS algorithm for system with bounded disturbance, Automatica 26 (1990), $599-606$.

[18] C.C. Lan and K.M. Lee, Generalized shooting method for analyzing compliant mechanisms, Proceedings of the 2005 IEEE International Conference on Robotics and Automation, Barcelona, Spain, (2005), 4309-4314.

[19] A. Wolf, J.B. Swift, H.L. Swinney and J.A. Vastano, Determining Lyapunov exponents from a time series, Physica D 16 (1985), $285-317$.

[20] L. Cao, Practical method for determining the minimum embedding dimension of a scalar time series, Physica D 110 (1997), 43-50.

[21] J. Theiler, S. Eubank, A. Longtin, B. Galdrikian and J. Doyne Farmer, Testing for nonlinearity in time series, the method of surrogate data, Physica D 58(1992), 77-94. 

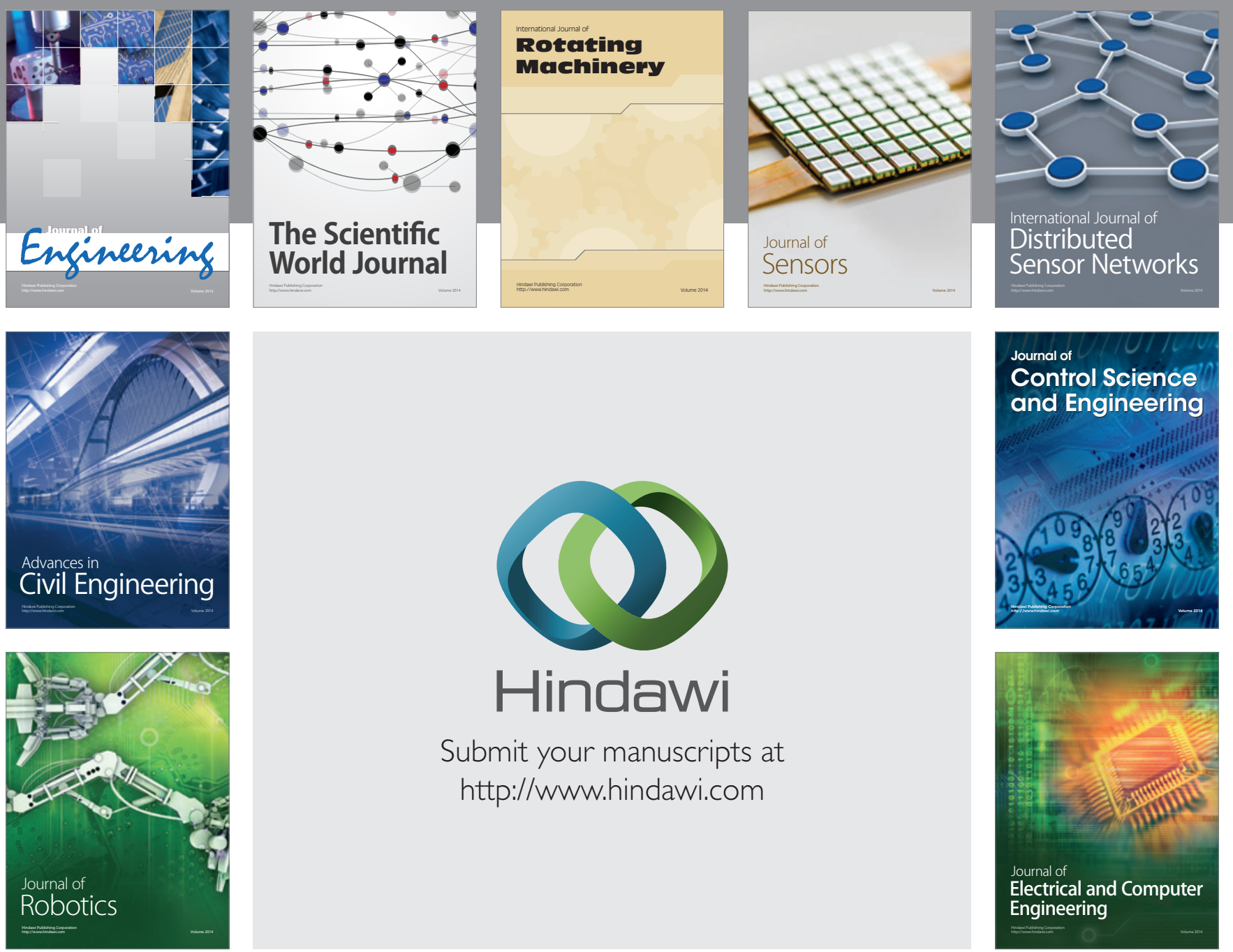

Submit your manuscripts at

http://www.hindawi.com
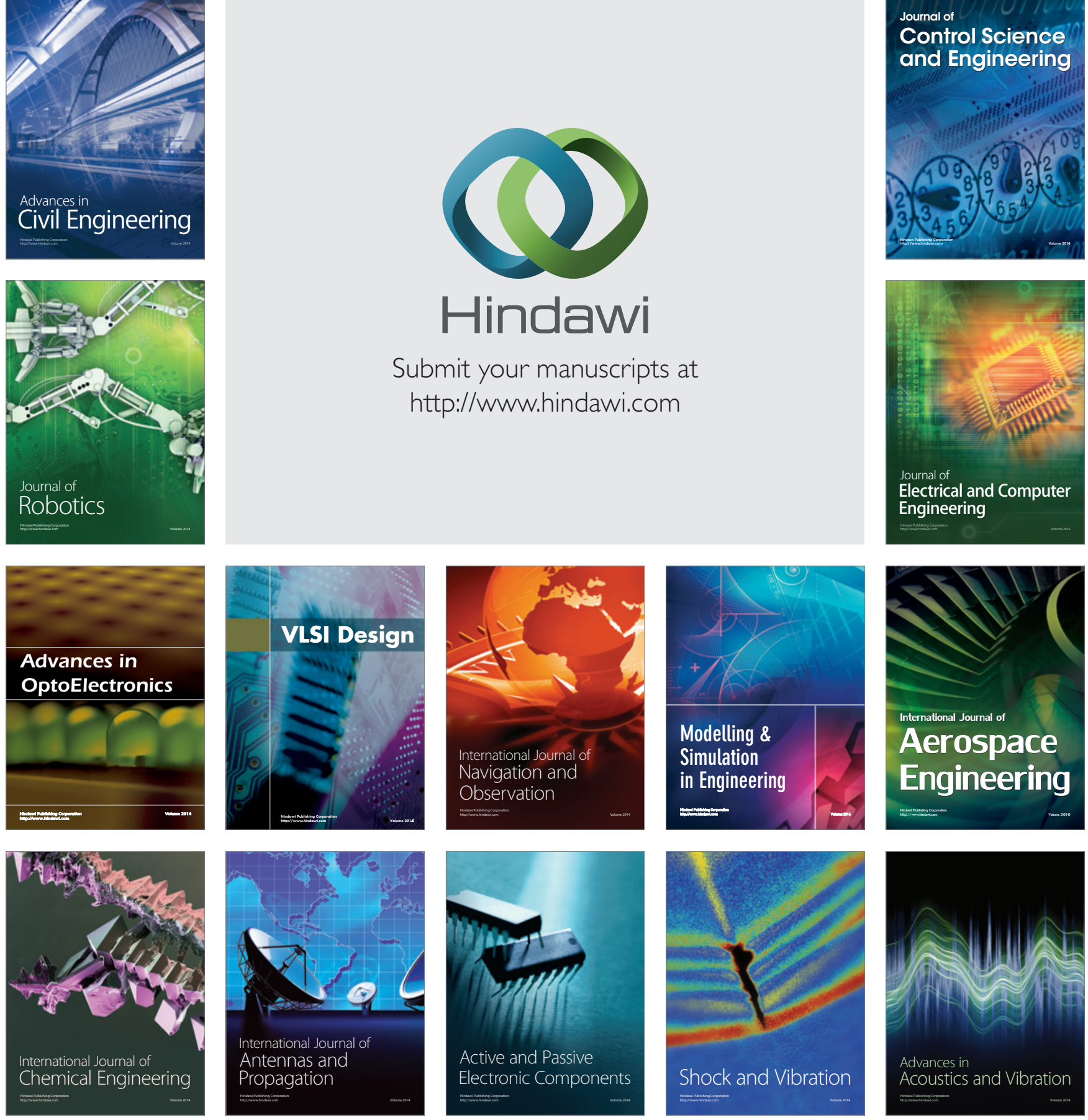